COMORBIDITY OF CHRONIC PAIN AND MENTAL HEALTH DISORDERS: THE BIOPSY CHOSOCIAL PERSPECTIVE*

\author{
Robert J. Gatchel, Ph.D., ABPP
}

* The writing of this article was supported in part by Grants No. 5R01 MH046452, 2K02

MH1107 and 5R01 DE010713 (from the National Institutes of Health) and Grant No.

DAMD17-03-1-0055 (from the Department of Defense).

The author would like to thank Dennis Turk for his careful reading of this manuscript To be presented at the Annual Meeting of the American Psychological Association,

Honolulu, Hawaii, July 29, 2004-Distinguished Professional Contributions to Applied Research Award for 2004.

Corresponding Author: $\quad$ Robert J. Gatchel, Ph.D., ABPP

Professor and Chairman

Department of Psychology, College of Science

The University of Texas at Arlington

Box 19528, 313 Life Science Bldg.

Arlington, TX 76019-0528

817.272.2541 (voice); 817.272.2364 (fax)

gatchel@uta.edu

RUNNING HEAD: The Biopsychosocial Perspective 


\begin{abstract}
We are entering an exciting period in mental and physical health research, resulting from a paradigm shift away from an outdated biomedical reductionism approach, to a more comprehensive biopsychosocial model, which emphasizes the unique interactions among biological, psychological and social factors required to better understand health and illness. This biopsychosocial perspective is important in evaluating the comorbidity of mental and physical health problems. Psychiatric and medical pathologies interface prominently in pain disorders. Important topics in the biopsychosocial approach to comorbid chronic mental and physical health disorders, focusing primarily on pain, are presented. Though this biopsychosocial model has produced dramatic advances in health psychology over the past two decades, important challenges to moving the field forward still remain.
\end{abstract}


The comorbidity of mental and physical health problems is well documented, especially when illness becomes chronic (e.g., Dersh, Polatin, \& Gatchel, 2002). Research has demonstrated that physical and psychological symptoms increase together, with a correlation of 0.5 between psychological distress scales and physical symptom checklists (Watson \& Pennebaker, 1989). Kroenke and colleagues (1994) have also reported that patients with anxiety or depressive disorders have more physical symptoms, and that as the number of physical symptoms increases so does the likelihood of an anxiety or depressive disorder. This holds true for symptoms with and without a diagnosed etiology. For example, Katon, Sullivan and Walker (2001) evaluated medical symptoms without clearly identified pathology (such as irritable bowel syndrome, fibromyalgia, headache, etc.), and noted a close association with psychiatric disorders such as panic disorder, major depression and somatization disorder. In a larger scale study by the World Health Organization, Gureje, Simon and Von Korff (2001) assessed 5,438 patients from 15 primary care sites and 14 countries. Of the $22 \%$ of patients who reported persistent pain for more than 6 months, there was a 4-fold increase in associated anxiety or depressive disorders. These relationships were consistent across cultures. Katon, Von Korff, Lin and colleagues (1990) found that, of the top $10 \%$ high utilizers of medical care, one-half carried a psychiatric diagnosis, such as depressive disorder (40\%), generalized anxiety disorder (22\%), panic disorder $(12 \%)$ and alcohol abuse (5\%).

Nowhere do psychiatric and medical pathologies interface more prominently than in pain disorders. Therefore, pain will be the focus of the present article in my discussion of comorbidity issues. Indeed, in this country, pain is a pervasive medical problem: it accounts for more than $80 \%$ of all physician visits; it affects in excess of 50 million Americans; and it costs more than $\$ 70$ billion annually in health care costs and lost productivity. Each year, an estimated 
176,850 patients seek treatment in pain centers in the U.S. alone (Marketdata Enterprises, 1995). In addition to the pain and emotional suffering these patients experience, chronic pain presents enormous costs to society. Such costs include lost earnings, decreased productivity, and increased health care utilization expenses and disability benefits. One study calculated the annual cost of chronic low back pain to be between $\$ 20$ billion and $\$ 60$ billion, when measures such as lost productivity and social security disability insurance benefits were calculated along with treatment costs (Gatchel \& Mayer, 2000). Thus, the prevalence and cost of chronic pain is still a major health care problem in the U.S. Recently, several important organizations in the U.S. have developed new standards for the evaluation of pain. One such organization — the Joint Commission on Accreditation of Healthcare Organizations—now requires that physicians consider pain as the " $5^{\text {th }}$ Vital Sign" (added to the other vital signs of pulse, blood pressure, core temperature and respiration). As another example of this current interest in pain, Congress has designated 2001-2010 as the Decade of Pain Control and Research.

\section{THE BIOPSYCHOSOCIAL PERSPECTIVE}

The most promising comorbidity- and pain-related clinical research conducted thus far has embraced a biopsychosocial (BPS) perspective. The emergence of this BPS perspective of mental health and pain disorders has paralleled the evolution of scientific thought in medicine (Gatchel, 1999). During the Renaissance, increased scientific knowledge in the areas of anatomy, biology and physiology was accompanied by a biomedical reductionism or a "dualistic" viewpoint that mind and body function separately and independently. This perspective dominated medicine until quite recently, and affected our understanding of the relationships between mental health and pain. The gate control theory of pain introduced by Melzack and Wall (1965), however, began to highlight the potentially significant role that 
psychosocial factors play in the perception of pain. Pain is now viewed as a complex set of phenomena, rather than as a simple, specific or discrete entity. This view converged with a BPS approach to medicine initially introduced by Engel (1977)that emerged during the 1970s and 1980s. In the past, organic pain was viewed as different from "psychogenic" pain. The term "psychogenic" suggested that the pain was due to psychological causes only, and that it was not "real" pain because no specific organic basis could be found. This perspective hindered the development of effective psychiatric and pain management strategies. Today, fortunately, the DSM-IV does not list "psychogenic pain" as a diagnostic entity. The assessment or diagnosis of organically caused pain does not rule out the important role that psychosocial factors can play for any particular patient. The general term "Pain Disorder" is used, with subtypes coded according to the relative degree of psychological and/or medical conditions associated with it. The BPS model views physical disorders such as pain as the result of a dynamic interaction among physiologic, psychologic and social factors, which perpetuates and may worsen the clinical presentation. Each individual experiences pain uniquely. A range of psychological and socioeconomic factors can interact with physical pathology to modulate patients' report of symptoms and subsequent disability. It should also be pointed out that, for medical conditions in which pain may not be the predominant symptom, this same BPS approach for better understanding and treating comorbid mental-physical interactions has been reviewed by Gatchel and Oordt (2003) for a number of prevalent illnesses — diabetes mellitus, hypertension and other cardiovascular disorders, asthma and gastrointestinal disorders.

Disease versus Illness

"To heal does not necessarily imply to cure. It can simply mean helping to achieve a way of life compatible with their individual aspirations - to restore their freedom to make choices- 
even in the presence of continuing disease." --Rene Dubos (1978)

This BPS model focuses on both disease and illness, with illness being viewed as a complex interaction of biological, psychological and social variables. According to Turk and Monarch, (2002), disease is defined as "an objective biological event" involving the disruption of specific body structures or organ systems caused by either anatomical, pathological or physiological changes. Illness, in contrast, is generally defined as a "subjective experience or self-attribution" that a disease is present. Thus, illness refers to how a sick individual and members of his or her family live with, and respond to, symptoms and disability. This distinction between disease and illness is analogous to the distinction made between pain and nociception. Nociception involves the stimulation of nerves that convey information about tissue damage to the brain. Pain, on the other hand, is a more subjective perception that is the result of the transduction, transmission and modulation of sensory input. This input may be filtered through a person's genetic composition, prior learning history, current psychological status, and sociocultural influences. Loeser (1982) originally formulated a model outlining four dimensions associated with the concept of pain (see Figure 1): the aforementioned dimensions of nociception and pain, as well as suffering (the emotional responses that are triggered by nociception or some other aversive event associated with it, such as fear or depression), and pain

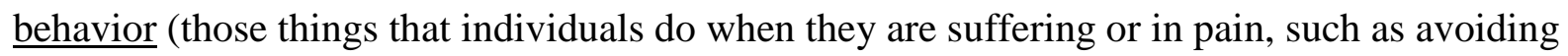
activities/exercise for fear of re-injury).

\section{INSERT FIGURE 1 ABOUT HERE}

Waddell (1987) subsequently pointed out that pain cannot be comprehensively assessed without a full understanding of the individual who is exposed to the nociception. He also made a 
comparison of Loeser's model of pain with an early BPS model of illness originally proposed by Engel (1977, Figure 1). Such a BPS model focuses primarily on illness. With this perspective, a diversity in pain or illness expression (including its severity, duration and psychosocial consequences) can be expected. The inter-relationships among biological changes, psychological status, and the sociocultural context all need to be considered in fully understanding the pain patient's perception and response to illness. Any model or treatment approach that focuses on only one of these core sets of factors will be incomplete.

\section{$\underline{\text { Biopsychosocial Interactions }}$}

Ray (2004) provided an excellent overview of mind-body relationships, and how social and behavioral factors can act on the brain to influence health, illness, and even death. In an earlier influential review, Cohen and Rodriguez (1995) pointed out important pathways linking mental and physical illness. In our own clinical research, we take such an interactive perspective, which recognizes the important afferent and efferent feedback between biological and psychological systems, as well as the effects of social mediators. Figure 2 presents a conceptual model of the BPS interactive processes involved in health and illness. We view pain as not purely a perceptual phenomenon,

\section{INSERT FIGURE 2 ABOUT HERE}

but the injury that has caused the pain also disrupts the body's homeostatic regulation systems which, in turn, produce stress and the initiation of complex programs to restore homeostasis. Melzack (1999) argues that recognizing the role of the stress system in pain processes significantly broadens the conceptualization of chronic pain and our ability to understand it. Chronic pain is a stressor that will "tax" the stress system. Prolonged activation of the stress- 
regulation systems will ultimately generate breakdowns of muscle, bone and neural tissue that, in turn, will cause more pain and produce a vicious cycle of pain-stress- reactivity. One important measure of the above pain-stress cycle is cortisol. As Melzack (1999) points out, along with the activation of the sympathetic nervous system, "cortisol sets the stage for the stress response...cortisol plays a central role because it is responsible for producing and maintaining high levels of glucose for the response. At the same time, cortisol is potentially a highly destructive substance because, to ensure a high level of glucose, it breaks down the protein in muscle and inhibits the ongoing replacement of calcium in bone." Indeed, cortisol is the main hormonal product of the hypothalamic-pituitary-adrenal (HPA) axis in humans. Although increased cortisol secretion is considered an adaptive response of the organism when stressed (for purposes of energy mobilization), prolonged secretion can lead to negative effects such as muscle atrophy, impairment of growth and tissue repair, immune system suppression, etc. Melzack suggests that cortisol will serve as a good marker of the degree of stress that should closely parallel the development of chronic pain. McEwen (1998) also highlights the importance of evaluating cortisol pattern dysregulation under conditions of allostatic load increases due to stress. HPA axis underlying mechanisms may therefore help to explain individual differences in stress and pain, as well as other medical conditions such as fibromyalgia.

We are also starting to develop an even broader view of BPS mechanisms in pain by using new brain imaging technology. This technology has not yet been systematically applied to the investigation of comorbidity issues in human subjects. One of the most popular and powerful imaging techniques now available is functional Magnetic Resonance Imaging (fMRI), which uses high-powered and rapidly oscillating magnetic-field gradients in order to detect changes in brain functioning (e.g., Rosenzweig, Breedlove, \& Leiman, 2002). It can create images of the 
brain which detect activities in its different parts, and it has advantages over earlier imaging techniques' spatial and temporal resolution, while not requiring patients to be injected with any substance such as radioactive material. A number of studies have demonstrated the effective use of fMRI in cortical regions and subcortical structures involved in pain processing (e.g., Bingel et al., 2002; Coghill, McHaffie, \& Yen, 2003). In addition, brain imaging studies have been used to investigate brain networks involved in placebo and opioid analgesia, as well as the basic functional neuroanatomy of the placebo effect as related to psychopharmacological response in depressed patients. Thus, imaging may provide a powerful new technique to help document the impact of different therapies.

\section{BIOPSYCHOSOCIAL, INTERDISCIPLINARY TREATMENT}

Now turning to treatment issues, the most promising work on pain conducted thus far has embraced a BPS, interdisciplinary approach in which the mental health needs of patients require careful evaluation and treatment, along with the concurrent physical pain problem. The treatment effectiveness of this approach to pain has consistently demonstrated the heuristic value of the model. Patients with chronic pain are at increased risk for depression, suicide, and sleep disorders. As pain becomes more chronic, emotional factors play an increasingly dominant role in the maintenance of dysfunction and suffering. Affective disorders, anxiety disorders, and substance abuse disorders are the three major psychiatric concomitants of chronic pain (Dersh et al., 2002). The significance of psychopathology in pain comorbidity is further evidenced by the potentially common pathogenetic mechanisms involved in psychiatric disorders, such as depression and pain (Polatin, 1991). Both nociceptive and affective pathways coincide anatomically. Furthermore, norepinephrine and serotonin, the two neurotransmitters most implicated in the pathophysiology of mood disorders, are also involved in the pain process. 
There have been a number of reviews that have documented the clinical effectiveness of such interdisciplinary treatment of chronic pain patients (e.g., Bendix et al., 1996; Gatchel, 1999; Okifuji, 2003). These programs are needed for chronic pain patients who have complex needs and requirements. One variant of such programs-functional restoration (FR)-has been comprehensively described in detail in a number of publications (e.g., Mayer \& Gatchel, 1988; Mayer et al., 1987; Mayer \& Polatin, 2000). Research has shown that the FR program is associated with substantive improvement in various important societal outcome measures (e.g., return to work and resolution of outstanding medical issues) in chronically disabled patients with spinal disorders in both 1-year follow-up studies (e.g., Hazard et al., 1989; Mayer et al., 1985), as well as a 2-year follow-up study (Mayer et al., 1987). In the 2-year follow-up study by Mayer et al., $87 \%$ of the FR group was actively working at 2 years post-treatment, as compared to only $41 \%$ of a non-treatment comparison group. Moreover, about twice as many of the comparison group patients had both additional spine surgery and unsettled workers' compensation litigation relative to the treatment group. The comparison group continued with approximately a 5-times higher rate of patient visits to health professionals and had higher rates of recurrence or re-injury. Thus, the results demonstrate the striking impact that an FR program can have on these important outcome measures in a chronic group consisting primarily of workers' compensation cases (traditionally the most difficult cases to successfully treat).

Finally, it should be noted that the original FR program was independently replicated by others in this country, as well as by investigators in Denmark, France, Germany, and Canada. The fact that different clinical treatment teams, functioning in different states and different countries, with markedly different economic/social conditions and workers' compensation systems, produced comparable outcome results speaks highly for the robustness of the research 
findings and utility, as well as the fidelity, of this FR approach. Gatchel and Turk (1999) and Turk (2002) have reviewed both the therapeutic- and cost-effectiveness of such programs.

\section{ETIOLOGIC ISSUES}

The success of interdisciplinary therapies incorporating a cognitive-behavioral orientation in improving the clinical status of chronic pain patients (Gatchel \& Turk, 1996) further attests to the major role that mental health issues play in chronic pain. Important questions, though, still remain. How are mental disorders exacerbated by pain and, conversely, how does a predisposition toward a psychiatric disorder affect the experience of pain and the evolution of chronic disability? What are the underlying neuropathways? We now also need a strong mental health life-span research emphasis. Chronic physical and mental health problems have become significant for the elderly, and the prevalence of such conditions can be expected to increase with the aging population. Individuals 50 years of age and older are twice as likely to have been diagnosed with chronic pain. Currently, there are approximately 35 million Americans age 65 years or older, accounting for $12.4 \%$ of the total population. By the year 2030 , it is projected that about $20 \%$ of the population will be 65 years or older (U.S. Census Bureau, 2000). Awareness of these population trends contributes to increased concern about health care issues among older adults, including mental health and pain problems. There has not yet been an organized focus on utilizing lifespan development approaches to the study of comorbid psychiatric and pain disorders.

Related to the above developmental issue, I have earlier presented a broad conceptual model of the transition from acute to chronic pain (Gatchel, 1996). More recent data from my laboratory further support the utility of this model (Gatchel \& Dersh, 2002). This model has proven useful in developing pain management strategies. It proposes 3 stages that may be 
involved in the transition of acute low back pain (LBP) into chronic LBP disability and accompanying psychosocial distress. Stage 1 is associated with emotional reactions, such as fear, anxiety, etc., due to the perception of pain during the acute phase. Pain or hurt is usually associated with harm, and so there is a natural emotional reaction to the potential for physical harm. If the pain persists past a reasonable acute period of time ( $2-4$ months), this leads to $\underline{\text { Stage } 2}$, which is associated with a wider array of psychological reactions and problems, such as learned helplessness-depression, distress-anger, somatization, etc., that result from the now more chronic nature of pain. The form these problems take will depend upon the premorbid or preexisting psychosocial characteristics of the individual, as well as current socioeconomic conditions. Thus, for a person with a premorbid problem with depression who is seriously affected economically by loss of a job due to pain, depressive symptomatology may be exacerbated during this Stage. Similarly, a significant personality disorder may begin to severely hamper a person's ability to cope with the stress of chronic pain. This model does not propose that there is one primary preexisting "pain personality," but assumes a general non-specificity in terms of the relationship between personality-psychosocial problems and pain. This is in keeping with research that has not found any such consistent personality syndrome. Thus, it is assumed that certain predisposing psychosocial characteristics differ from one patient to the next, and may be exacerbated by the stress of attempting to cope with pain.

The above conceptual model proposes that as the "layer" of behavioral/psychological problems persists, it progresses into $\underline{\text { Stage } 3}$ which is viewed as the acceptance or adoption of a "sick role" (in Engel's, 1977 previously presented model) during which patients are excused from their normal responsibilities and social obligations. This may become a potent reinforcer for not becoming "healthy." The medical and psychological "disabilities" or "abnormal illness 
behaviors" (Pilowsky, 1978)are consolidated during this phase. Research consistently

demonstrates the important psychological changes that occur as a pain patient progresses from the acute to more chronic phases. This model also proposes that superimposed on these three stages is what is known as the physical "deconditioning syndrome" (Mayer \& Gatchel, 1988). This refers to a significant decrease in physical capacity (strength, flexibility and endurance) due to disuse and the resultant atrophy of the injured area. There is usually a two-way pathway between the physical deconditioning and the above stages.

In summary, the medical condition of pain is quite prevalent, consumes a high proportion of the health care dollar, and will increase with the "graying of America." It has a high comorbidity with mental health problems. Scientific research is just beginning to broaden our understanding of this diathesis. My ongoing research will continue to investigate the etiology, prevention and treatment of mental health problems in patients with chronic pain, and will focus on furthering our understanding of the development and nature of chronic pain when accompanied by psychiatric disorders, which will be discussed next.

\section{PSYCHOPATHOLOGY AND CHRONIC PAIN DISORDERS}

In conducting clinical research, it became quite apparent that patients with chronic pain (as well as other chronic medical illnesses) commonly exhibit increased levels of emotional distress and psychopathology that interfere with effective treatment. As a result, we started to conduct a series of studies to evaluate the prevalence of DSM Axis I and Axis II diagnoses of pain patients, derived from a structured interview format (the Structured Clinical Interview for DSM-IV; SCID). One of our initial studies investigating the nature of the relationship between chronic pain and psychopathology assessed 200 chronic LBP patients for current and lifetime psychiatric syndromes (Polatin, P. B., Kinney, R. K., Gatchel, R. J., Lillo, E., \& Mayer, T. G., 
1993). Even when the somewhat controversial category of somatoform pain disorder was excluded, $77 \%$ of patients met lifetime diagnostic criteria, and 59\% demonstrated current symptoms, for at least one psychiatric diagnosis. The most common were major depression, substance abuse, and anxiety disorders. In addition, $51 \%$ met criteria for at least one personality disorder. All of the prevalences were significantly greater than base rates for the general population. These are strikingly high rates of psychopathology in this chronic pain population, and are comparable to rates that have been reported in other studies. Subsequently, we found comparably high rates of psychopathology in other chronic pain disorders, such as temporomandibular disorder (TMD, Gatchel, Garofalo, Ellis, \& Holt, 1996), and upper extremity disorders such as carpal tunnel syndrome (Mathis, Gatchel, Polatin, Boulas, \& Kinney, 1994).

The Polatin et al. (1993) study also found that, of those patients with a positive lifetime history of psychiatric disorders, $54 \%$ of those with depression, $94 \%$ of those with substance abuse, and $95 \%$ of those with anxiety disorders had apparently experienced these syndromes before the onset of their LBP. These were the first results to suggest that certain psychiatric syndromes appear to precede chronic LBP (substance abuse and anxiety disorders), whereas others (specifically, major depression) develop either before or after the onset of their LBP. Depression was demonstrably high in chronic LBP patients, and patients appeared to be divided equally between those who had depression before the onset of pain and those in whom depression developed after the onset of pain. This was one of the first studies to evaluate an important aspect of the "chicken and/or egg" question: what comes first, the pain or the psychopathology? We are continuing to evaluate the nature of the relationship between the two variables.

In another study, which led to the development of a prediction model to be used in our 
future studies, Gatchel, Polatin and Mayer (1995) found evidence of a "psychosocial disability factor" in LBP. In this study, 421 patients presenting with acute LBP complaints of less than 6 months were systematically evaluated with a comprehensive psychosocial assessment battery. Then, 1 year after the initial evaluation, all patients were contacted to participate in a structured telephone interview to document return-to-work status. The responses generated a logistic regression model which found that the following array of variables correctly classified $90.7 \%$ of the cases in terms of work status at 1 year: self-reported pain and disability scores, scores on Scale 3 of the MMPI, workers' compensation/personal injury insurance status, and gender. Thus, these results revealed the presence of a "psychosocial disability factor" associated with those injured workers most likely to develop chronic LBP disability problems at 1 year. We can now identify those acute LBP patients who may require early intervention to prevent development of chronic disability. This has formed the basis for a subsequent early intervention translational research with "high-risk" patients. There were also no significant differences between the return-to-work and no-return-to-work groups in terms of physician-rated severity of the initial back injury or in the physical demands of the jobs to which the patients had to return.

Such results again highlight the fact that chronic pain disability reflects not only the presence of some physical symptomatology, but that psychosocial characteristics make a significant contribution in determining which injured workers may develop chronic LBP disability. In fact, many have argued that only a small amount of the total disability phenomenon in people complaining of LBP can be attributed to physical impairment. Indeed, most cases of LBP are ill defined and physically unverifiable, and are often classified as "soft tissue injuries" that cannot be visualized or verified on physical examination. Even the correlation between radiographic-documented disc-space narrowing and disc rupture level including disc herniation 
is less than 50\%. Moreover, an MRI study by Jensen, Brandt-Zawadzki, Obuchowski, et al.

(1994) found significant spinal abnormalities in patients not experiencing LBP.

\section{OTHER COMORBIDITY CLINICAL RESEARCH ISSUES}

Along with our research on psychopathology and chronic pain disorders, we are also involved in the development of treatment outcomes research of interdisciplinary approaches to other chronic comorbid mental health and pain disorders. As previously noted, interdisciplinary treatment emphasizes the importance of addressing the complex needs of chronic pain patients, including their psychosocial needs. The first truly effective interdisciplinary approach to chronic low back pain patients_-FR — was developed by Mayer and Gatchel (1988), which subsequently generated numerous studies documenting its effectiveness. It has also been shown to be effective for cervical spine disorders, upper extremity disorders, as well as heterogeneous pain disorders. In all of our studies, we have been interested in the management of the comorbid mental health problems experienced by patients undergoing treatment, and whether it would limit successful rehabilitation. In one such study, we used the SCID to assess the prevalence of current and lifetime DSM diagnoses in a sample of chronic LBP patients beginning an intensive 3-week FR program (Gatchel, Polatin, Mayer, \& Garcy, 1994). These patients were then followed over time, with treatment outcome being defined as return-to-work status one year after program completion. Despite high rates of Axis I and Axis II psychiatric disorders in this sample, neither type nor degree of psychopathology was found to be predictive of a patient's ability to successfully return to work. An equally interesting series of results showed that elevated rates of psychopathology significantly decreased following the FR treatment of chronic LBP patients (Vittengl, Clark, Owen-Salters, \& Gatchel, 1999).

\section{Patient Heterogeneity and Response to Treatment}


We are also beginning to evaluate variables that may predict which patients respond best to such programs (see Gatchel \& Epker, 1999). As noted by Turk and Okifuji (1998), one must avoid the assumption of "pain-patient homogeneity" in terms of response to treatment. There may be many individual differences or heterogeneity in such responses. Individuals with the same medical diagnosis may vary greatly in their response to their symptoms. Turk and colleagues, for example, have revealed that patients with diseases and syndromes as varied as back pain, headache, and metastatic cancer may display comparable adaptation patterns, whereas patients with the same diagnosis may actually show great variability in their degree of disability (e.g., Turk, Okifuji, Sinclair, \& Starz, 1998). As Turk and Gatchel (1999) have indicated, the traditional approach of "lumping" patients with the same medical diagnosis or set of symptoms together (e.g., fibromyalgia, LBP, TMD), and then to treat them all the same way, is not appropriate. That is because many of these common diagnoses are relatively gross categories, and there may be unique individual BPS differences of patients who fall under these generic diagnoses. Thus, some patients may respond quite positively to a certain treatment, whereas others may actually show no improvement at all. Therefore, it is becoming more important to match a particular intervention to specific patient characteristics. The "pain patient homogeneity" myth must be debunked, and patient differences need to be taken into account in order to tailor the appropriate treatment program.

A number of studies have already demonstrated that patients classified into different subgroups based on their behavioral and psychosocial characteristics responded differentially to identical treatments (Turk, 2002). This has been fairly consistently observed across different types of pain syndromes. The differences in the psychosocial profiles displayed by patients has led to attempts to categorize different subgroups of patients and then to evaluate differential 
response to a treatment. For example, several outcome studies have demonstrated the effective use of the Multidimensional Pain Inventory (MPI) as one way to categorize subgroups of patients (as adaptive copers, dysfunctional, or interpersonally distressed). Adaptive Coper patients report a high level of social support and relatively low level of pain and perceived interference with their lives. In addition, they usually report relatively high levels of activity despite their pain, and often respond well to pain management procedures. In contrast, dysfunctional profile patients tend to perceive the severity of their pain to be high and to report that pain interferes with much of their lives. They also report a high degree of psychological distress because of their pain and, as a result, usually report low levels of activity. Interpersonally distressed patients are similar to dysfunctional profile patients, but they also perceive that their significant others are not very understanding about their condition. They, therefore, think that they have no good social support to help them with their pain behavior problems.

Assessment of such MPI profiles will help to "tailor" the needs for treatment strategies to account for the different psychosocial characteristics of patients. For example, patients with an interpersonally distressed profile may need additional clinical attention addressing interpersonal skills to perform effectively in a group-oriented treatment program. Pain patients with dysfunctional and interpersonally distressed profiles display more indications of acute and chronic personality differences, relative to adaptive coper profile patients, and they would therefore require more clinical management (Etscheidt, Steiger, \& Braverman, 1995). Such additional attention, however, would not necessarily be essential for adaptive coper profile patients. These studies support the notion that, because patients' responses to treatment differ as a function of their psychosocial coping profiles, then specific treatment modalities are more likely to be better suited than others for each profile. An important issue for future clinical 
research is whether there are other types of BPS profiles that are more or less responsive to different treatment modalities.

Early Intervention with High-Risk Acute Pain Patients

As reviewed earlier, our most recent research has focused on the use of the empirically developed "high-risk" acute patient profile associated with the development of chronic disability problems. Our first one-year prospective outcome study (Gatchel et al., 2003), clearly revealed that high-risk acute LBP patients who received early intervention displayed significantly greater improvements in psychosocial functioning, pain levels, medication use, health care utilization and occupational outcomes. Greater cost savings were also found. We are currently conducting a similar study with acute TMD patients employing an abbreviated version of functional restoration, developed specifically for these research programs. Again, it involves an interdisciplinary team approach, and is based upon the assumption that almost all patients suffering from comorbid mental health and pain disability can be returned to a productive lifestyle through appropriate medical care/physical reconditioning and psychosocial interventions such as coping skills training. This FR is accomplished through an aggressive, individualized psychosocial and physical reconditioning program. Treatment is initially guided by quantified measurement of function, allowing the reconditioning to proceed safely, but providing quantifiable documentation of compliance, effort and eventual success. Psychosocial issues and return-to-work issues are simultaneously addressed. We have found that such issues can be effectively dealt with using psychosocial approaches (cf Turk \& Gatchel, 2002)

\section{SUMMARY AND CONCLUSIONS}

We are entering an exciting period in mental and physical health research, resulting from a major paradigm shift away from an outdated biomedical reductionism approach, to a more 
heuristic and comprehensive BPS model which emphasizes the unique interactions among biological, psychological and social factors that need to be taken into account to better understand health and illness. Suls and Rothman (2004, p. 119) have recently reviewed the evolution of the BPS model, and noted how the reciprocal relationship of the biological, psychological and social processes has stimulated dramatic advances in health psychology over the past two decades. As they point out: "As a guiding framework, the BPS model has proven remarkably successful as it has enabled health psychologists to be at the forefront of efforts to forge a multilevel, multisystems approach to human functioning. However, considerable, perhaps even daunting challenges remain as models are needed that specify the processes that connect the biological, psychological, and social systems." Another major reason for the now heightened acceptance of the BPS model has been the increase in prevalence of chronic medical illnesses in this country, which can be expected to continue to rise. Chronic medical illnesses most often are accompanied by comorbid mental health problems, thus necessitating the use of a BPS approach to assessment and intervention programs for such comorbid chronic illnesses. As reviewed in this article, the BPS approach to one such prevalent comorbid chronic medical problem - pain — has been shown to be quite effective, and it will hopefully lead to further breakthroughs in the areas of etiology, assessment, treatment and prevention. Of course, the major paradigm shift to the BPS model is still in its infancy stage, and new developments can be expected in the future. Our major task as behavioral scientists will be to ensure that its continued evolution occurs in a scientifically rigorous manner, while also keeping our eyes on the "bulls eye"- the systematic translation of this new BPS approach to comorbid disorders into the clinical arena where it can be further validated. With great opportunity comes equally great responsibility for progress in this exciting new era for psychologists: 
The Biopsychosocial Perspective

"The best preparation for tomorrow is to do today's work superbly well." --Sir William Osler 


\section{REFERENCES}

Bendix, A. E., Bendix, T., Vaegter, K., Lund, C., Frolund, L., \& Holm, L. (1996). Multidisciplinary intensive treatment for chronic low back pain: A randomized, prospective study. Cleveland Clinic Journal of Medicine, 63, 62-69.

Bingel, V., Quante, M., Knab, R., Bromm, B., Weiller, C., \& Buchel, C. (2002). Subcortical structures involved in pain processing: Evidence from single trial fMRI. Pain, 99, 313321.

Coghill, R. C., McHaffie, J. G., \& Yen, Y. F. (2003). Neural correlates of interindividual differences in the subjective experience of pain. PNAS, 100(14), 8538-8542.

Cohen, S., \& Rodriguez, M. S. (1995). Pathways linking affective disturbances and physical disorders. Health Psychology, 14, 371-373.

Dersh, J., Polatin, P., \& Gatchel, R. (2002). Chronic pain and psychopathology: Research findings and theoretical considerations. Psychosomatic Medicine, 64, 773-786.

Engel, G. L. (1977). The need for a new medical model: A challenge for biomedicine. Science, 196(4286), 129-136.

Etscheidt, M. A., Steiger, H. G., \& Braverman, B. (1995). Multidimensional pain inventory profile classifications and psychopathology. Journal of Consulting \& Clinical Psychology, 51, 29-36.

Gatchel, R. J. (1996). Psychological disorders and chronic pain: Cause and effect relationships. In R. J. Gatchel \& D. C. Turk (Eds.), Psychological Approaches to Pain Management: A Practitioner's Handbook (pp. 33-52). New York: Guilford. 
Gatchel, R. J. (1999). Perspectives on Pain: A Historical Overview. In R. J. Gatchel \& D. C. Turk (Eds.), Psychosocial Factors in Pain: Critical Perspectives (pp. 3-17). New York: Guilford Publications.

Gatchel, R. J., \& Dersh, J. (2002). Psychological Disorders and Chronic Pain: Are There Cause and Effect Relationships? In D. C. Turk \& R. J. Gatchel (Eds.), Psychological Approaches to Pain Management: A Practitioner's Handbook (2nd ed.). New York: Guilford.

Gatchel, R. J., \& Epker, J. T. (1999). Psychosocial predictors of chronic pain and response to treatment. In R. J. Gatchel \& D. C. Turk (Eds.), Psychosocial Factors in Pain: Critical Perspectives (pp. 412-434). New York: Guilford Publications, Inc.

Gatchel, R. J., Garofalo, J. P., Ellis, E., \& Holt, C. (1996). Major psychological disorders in acute and chronic TMD: An initial examination of the "chicken or egg" question. Journal of the American Dental Association, 127, 1365-1374.

Gatchel, R. J., \& Mayer, T. G. (2000). Occupational Musculoskeletal Disorders: Introduction and Overview of the Problem. In T. G. Mayer, R. J. Gatchel \& P. B. Polatin (Eds.), Occupational Musculoskeletal Disorders: Function, Outcomes, and Evidence (pp. 3-8). Philadelphia: Lippincott Williams \& Wilkins.

Gatchel, R. J., \& Oordt, M. S. (2003). Clinical Health Psychology and Primary Care: Practical Advice and Clinical Guidance for Successful Collaboration. Washington, DC: American Psychological Association.

Gatchel, R. J., Polatin, P. B., \& Mayer, T. G. (1995). The dominant role of psychosocial risk factors in the development of chronic low back pain disability. Spine, 20(24), 2702-2709. 
Gatchel, R. J., Polatin, P. B., Mayer, T. G., \& Garcy, P. D. (1994). Psychopathology and the rehabilitation of patients with chronic low back pain disability. Archives of Physical Medicine and Rehabilitation, 75, 666-670.

Gatchel, R. J., Polatin, P. B., Noe, C. E., Gardea, M. A., Pulliam, C., \& Thompson, J. (2003). Treatment- and cost-effectiveness of early intervention for acute low back pain patients: A one-year prospective study. Journal of Occupational Rehabilitation, 13, 1-9.

Gatchel, R. J., \& Turk, D. C. (1996). Psychological Approaches to Pain Management: A Practitioner's Handbook. New York: Guilford Publications, Inc.

Gatchel, R. J., \& Turk, D. C. (1999). Interdisciplinary treatment of chronic pain patients. In R. J. Gatchel \& D. C. Turk (Eds.), Psychosocial Factors in Pain: Critical Perspectives (pp. 435-444). New York: Guilford.

Gureje, O., Simon, G., \& Von Korff, M. (2001). A cross-national study of the course of persistent pain in primary care. PAIN, 92(1-2), 195-200.

Hazard, R. G., Fenwick, J. W., Kalisch, S. M., Redmond, J., Reeves, V., Reid, S., et al. (1989). Functional restoration with behavioral support: A one-year prospective study of patients with chronic low-back pain. Spine, 14, 157-161.

Jensen, M. C., Brant-Zawadzki, M. N., Obuchowski, N., Modic, M. T., Malkasian, D., \& Ross, J. S. (1994). Magnetic resonance imaging of the lumbar spine in people without back pain. The new England Journal of Medicine, 331, 69-73.

Katon, W., Sullivan, M., \& Walker, E. (2001). Medical symptoms without identified pathology: relationship to psychiatric disorders, childhood and adult trauma, and personality traits. Annals of Internal Medicine, 134(9 pt 2), 917-925. 
Katon, W., Von Korff, M., Lin, E., Lipscomb, P., Russo, J., Wagner, E., et al. (1990). Distressed high utilizers of medical care. DSM-III-R diagnoses and treatment needs. General Hospital Psychiatry, 12(6), 355-362.

Kroenke, K., Spitzer, R. L., \& Williams, J. B. (1994). Physical symptoms in primary care. Predictors of psychiatric disorders and functional impairment. Archives of Family Medicine, 3(9), 774-779.

Loeser, J. D. (1982). Concepts of Pain. In J. Stanton-Hicks \& R. Boaz (Eds.), Chronic Low Back Pain. New York: Raven Press.

Marketdata Enterprises. (1995). Chronic Pain Management Programs: A Market Analysis. Valley Stream, NY: Author.

Mathis, L. B., Gatchel, R. J., Polatin, P. B., Boulas, J., \& Kinney, R. (1994). Prevalence of psychopathology in carpal tunnel syndrome patients. Journal of Occupational Rehabilitation, 4, 199-210.

Mayer, T. G., \& Gatchel, R. J. (1988). Functional Restoration for Spinal Disorders: The Sports Medicine Approach. Philadelphia: Lea \& Febiger.

Mayer, T. G., Gatchel, R. J., Kishino, N., Keeley, J., Capra, P., Mayer, H., et al. (1985). Objective assessment of spine function following industrial injury: A prospective study with comparison group and one-year follow-up. Spine, 10, 482-493.

Mayer, T. G., Gatchel, R. J., Mayer, H., Kishino, N. D., Keeley, J., \& Mooney, V. A. (1987). A prospective two year study of functional restoration in industrial low back injury utilizing objective assessment. JAMA, 258, 1763-1767.

Mayer, T. G., \& Polatin, P. B. (2000). Tertiary nonoperative interdisciplinary programs: The functional restoration variant of the outpatient chronic pain management program. In T. 
G. Mayer, R. J. Gatchel \& P. B. Polatin (Eds.), Occupational Musculoskeletal Disorders: Function, Outcomes \& Evidence (pp. 639-649). Philadelphia: Lippincott, Williams \& Wilkins.

McEwen, B. S. (1998). Protective and damaging effects of stress mediators. New England Journal of Medicine, 338, 171-179.

Melzack, R. (1999). Pain and stress: A new perspective. In R. J. Gatchel \& D. C. Turk (Eds.), Psychosocial Factors in Pain: Critical Perspectives. New York: Guilford Publications, Inc.

Melzack, R., \& Wall, P. D. (1965). Pain mechanisms: A new theory. Science, 50, 971-979.

Okifuji, A. (2003). Interdisciplinary pain management with pain patients: Evidence for its effectiveness. Seminars in Pain Management, 1, 110-119.

Pilowsky, I. (1978). A general classification of abnormal illness behavior. British Journal of Medical Psychiatry, 51(2), 131-137.

Polatin, P. (1991). Predictors of low back pain. In A. White \& R. Anderson (Eds.), Conservative Care of Low Back Pain. Baltimore: Williams \& Wilkins.

Polatin, P. B., Kinney, R., Gatchel, R. J., Lillo, E., \& Mayer, T. G. (1993). Psychiatric Illness and Chronic Low Back Pain: The Mind and the Spine-Which Goes First? Spine, 18, 6671.

Polatin, P. B., Kinney, R. K., Gatchel, R. J., Lillo, E., \& Mayer, T. G. (1993). Psychiatric illness and chronic low-back pain. The mind and the spine--Which goes first? Spine, 18(1), 6671.

Ray, Q. (2004). How the mind hurts and heals the body. American Psychologist, 59, 29-40. 
Rosenzweig, M. R., Breedlove, S. M., \& Leiman, A. L. (2002). Biological Psychology. Sunderland, MA: Sinauer Associates.

Suls, J., \& Rothman, A. (2004). Evolution of the biopsychosocial model: Prospects and challenges for health psychology. Health Psychology, 23, 119-125.

Turk, D. C. (2002). Clinical effectiveness and cost effectiveness of treatment for patients with chronic pain. Clinical Journal of Pain, 18, 355-365.

Turk, D. C., \& Gatchel, R. J. (1999). Psychosocial factors and pain: Revolution and evolution. In R. J. Gatchel \& D. C. Turk (Eds.), Psychosocial Factors in Pain: Critical Perspectives. New York: Guilford.

Turk, D. C., \& Gatchel, R. J. (Eds.). (2002). Psychological Approaches to Pain Management: A Practitioner's Handbook (2nd ed.). New York: Guilford.

Turk, D. C., \& Monarch, E. S. (2002). Biopsychosocial perspective on chronic pain. In D. C. Turk \& R. J. Gatchel (Eds.), Psychological Approaches to Pain Management: A Practitioner's Handbook (2nd ed.). New York: Guilford.

Turk, D. C., \& Okifuji, A. (1998). Treatment of chronic pain patients: Clinical outcomes, costeffectiveness, and cost-benefits of multidisciplinary pain centers. Critical Reviews in Physical and Rehabilitation Medicine, 10(181-208).

Turk, D. C., Okifuji, A., Sinclair, J. D., \& Starz, T. W. (1998). Interdisciplinary treatment for fibromyalgia syndrome: Clinical and statistical significance. Arthritis Care \& Research, 11, 186-195.

U.S. Census Bureau. (2000). Population projections of the United States by age, sex, race, Hispanic origin, and nativity: 1999 to 2100. Washington, D.C.: U.S. Census Bureau. 
Vittengl, J., Clark, L., Owen-Salters, E., \& Gatchel, R. (1999). Diagnostic change and personality stability following functional restoration treatment in a chronic low back pain patient sample. Assessment, 6, 79-92.

Waddell, G. (1987). Clinical assessment of lumbar impairment. Clinical Orthopedic Related Research, 221, 110-120.

Watson, D., \& Pennebaker, J. (1989). Health complaints, stress, and distress: Exploring the central role of negative affectivity. Psychological Review, 96(2), 234-254. 
ENGEL'S

CONCEPTUAL MODEL OF ILLNESS

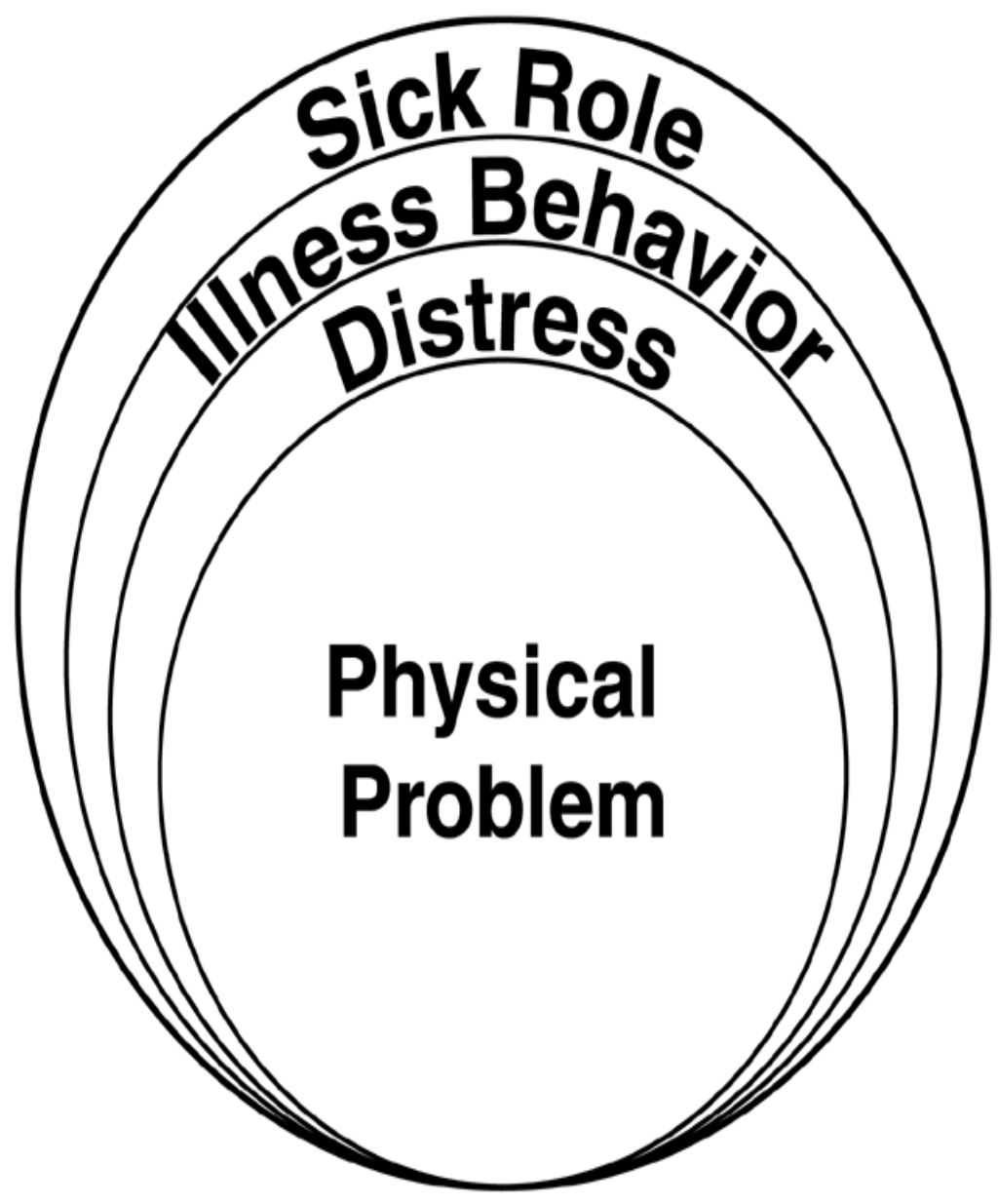

LOESER'S

CONCEPTUAL MODEL OF PAIN

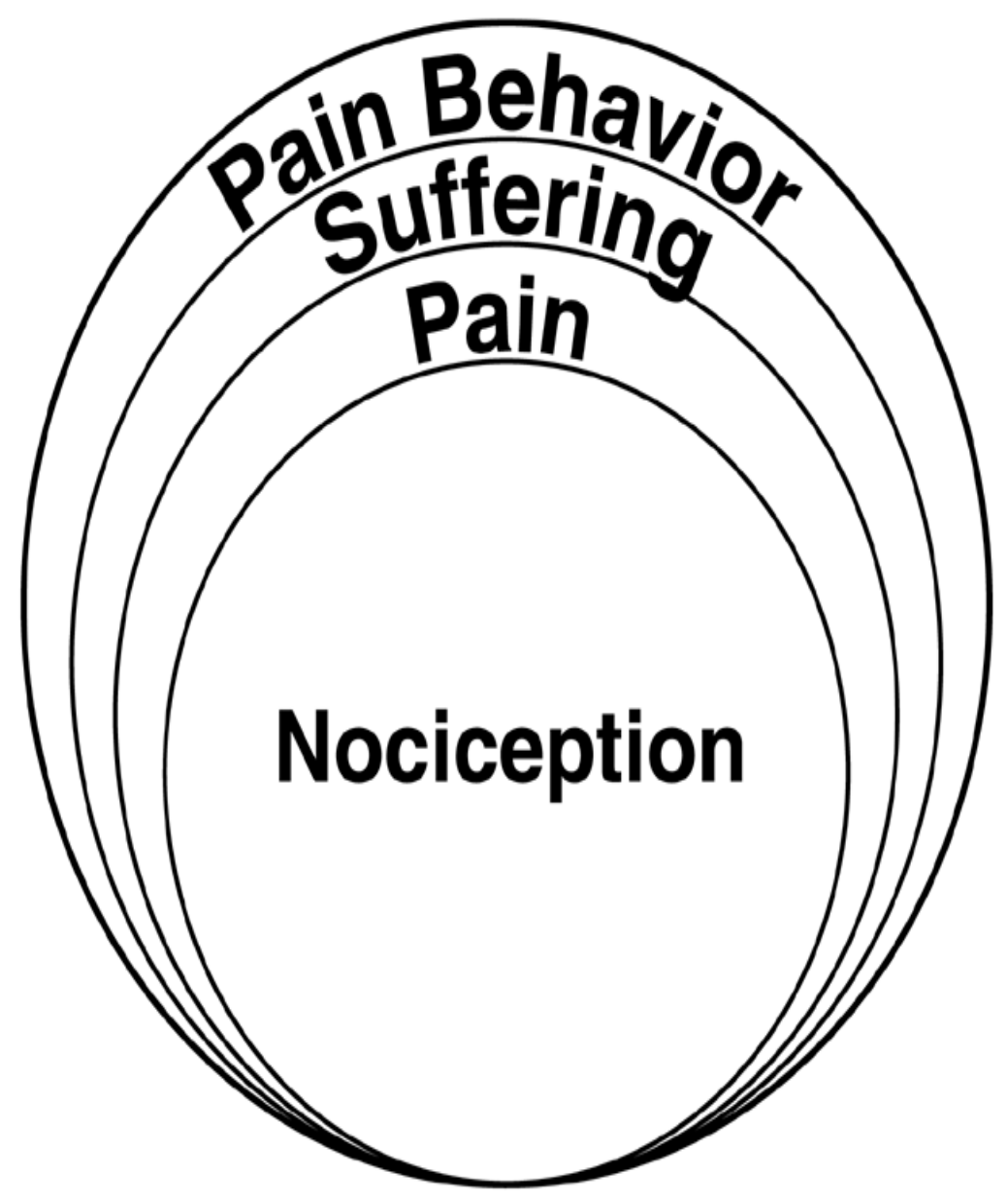

Figure 1: The biopsychosocial models of pain and illness 


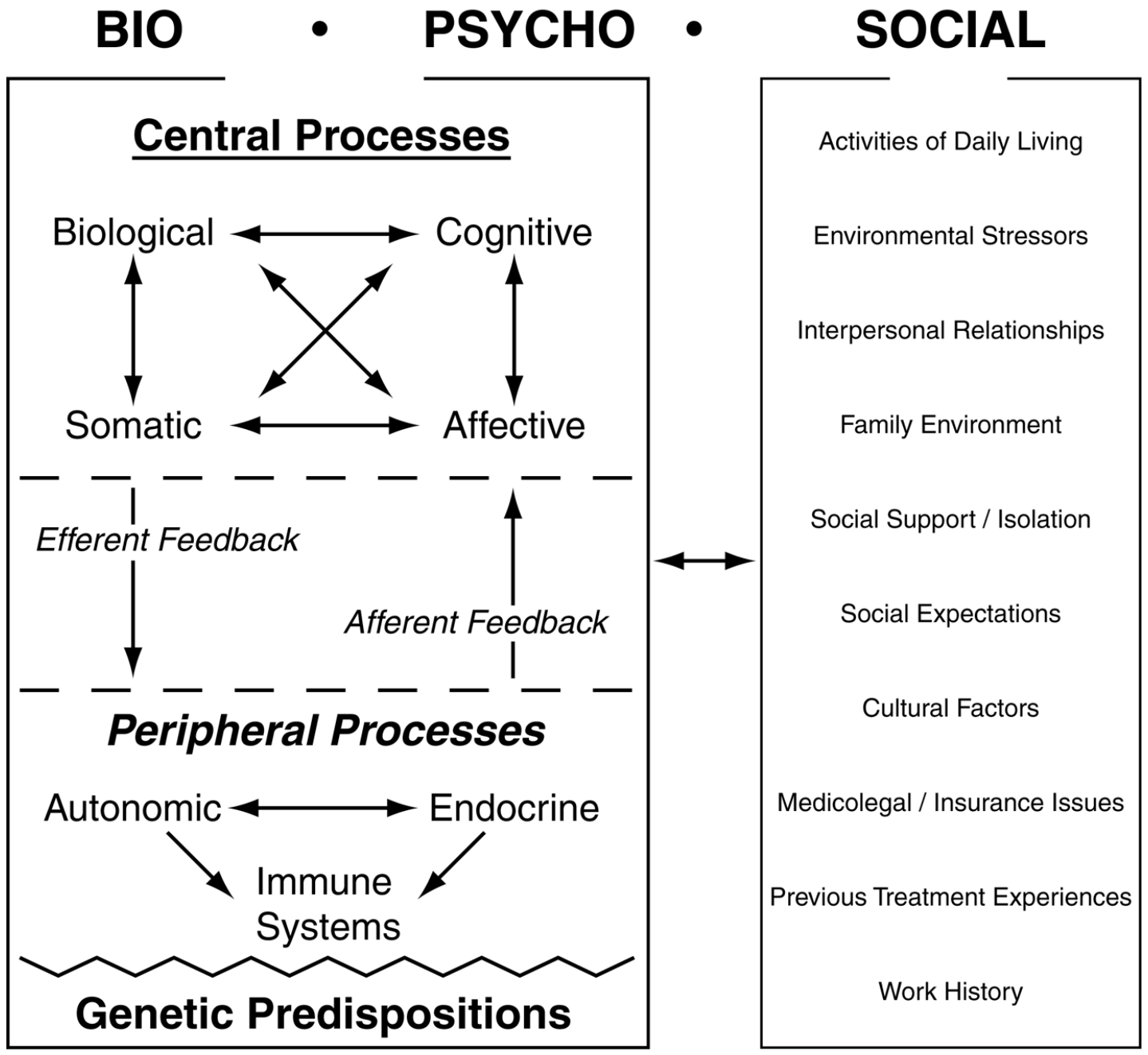

Figure 2. A conceptual model of the biopsychosocial interactive processes involved in health and fitness. 